\title{
Review: the case for antidepressants for treating depression in people with schizophrenia remains unproven
}

Whitehead C, Moss S, Cardno A, Lewis G. Antidepressants for the treatment of depression in people with schizophrenia: a systematic review. Psychol Med 2003;33:589-599.

\section{QUESTION: Are antidepressants effective for treating depression in people with schizophrenia?}

\section{Design}

Systematic review with meta-analysis.

\section{Data Sources}

ClinPsych, the Cochrane Library, the Cochrane Schizophrenia Group's Register of Trials, EMBASE and Medline were searched in December 2000; reference lists were hand searched. Drug companies and authors of reports published in the last 10 years were contacted.

\section{Study selection}

Eligible studies were randomised controlled trials comparing antidepressants versus placebo in people with schizophrenia or schizoaffective disorder, and a depressive episode. Studies that permitted concurrent medication with antipsychotics or anticholinergics were eligible.

\section{Data extraction}

Data were extracted on sample size, study design, participant characteristics, interventions and outcomes. The main outcome measures were recovery from depression (as defined by the primary studies), and the Hamilton depression rating scale.

\section{Main Results}

11 studies met the inclusion criteria. The studies were of generally poor quality and were small. Only some studies were suitable for meta-analysis. Meta-analysis of the 5 suitable trials showed that antidepressants significantly increased recovery from depression compared with placebo (absolute risk difference in favour of antidepressants $26 \%, 95 \%$ CI $10 \%$ to $42 \%$; NNT 4, 95\% CI 3 to 9 ). However, meta-analysis of the 6 studies reporting scores on the Hamilton depression rating scale found no significant difference between groups (standardised mean difference $-0.27,95 \% \mathrm{CI}-0.7$ to +0.2 ).

\section{Conclusions}

The meta-analysis found weak evidence to support a benefit of antidepressants in schizophrenia. However, this conclusion may be limited by publication bias and larger, more robust studies are needed.

\section{COMMENTARY}

Depression in schizophrenia is a common problem and is associated with a suicide rate of $10-15 \%$. Earlier reviews have found antidepressant treatment beneficial in select patient groups..$^{1-3}$ Levinson ${ }^{1}$ concluded that antidepressants are beneficial for treatment of depression in people with schizophrenia who are stable with respect to psychotic symptoms, whereas antipsychotics were more effective in people with acute psychosis.

The systematic review by Whitehead et al investigated the benefit of antidepressant medication in people with schizophrenia and comorbid depression. Strengths of this review include descriptions of the studies used for analysis with calculations of risk difference or effect sizes for each study. However, as the original trials were of limited quality in terms of size, heterogeneity, or did not report data, meta-analysis could only be performed on subsets of trials.

Although the systematic review included studies in people with different stages of schizophrenia, the small number of studies did not allow the use of antidepressants during different stages of schizophrenia to be assessed. Similarly, effects of typical and atypical medications could not be evaluated. This is of interest, since atypical antipsychotics appear more beneficial in improvement of anxiety and depression in people with schizophrenia. ${ }^{4}$ Most studies employed tricyclic antidepressants not considered first line at the present time, limiting relevance to practice today. Due to more selective effects on neurotransmitters, superior tolerability and safety, newer generation antidepressants may yield beneficial effects that were not found with the older medications.

This systematic review concludes that there is weak evidence for the effectiveness of antidepressant medication in people with schizophrenia and depression, however the literature reviewed was of such limited quality that positive results might be explained by publication bias.

Christian G Kohler, MD Neuropsychiatry Program Dept. of Psychiatry University of Pennsylvania Philadelphia, USA

1 Levinson DF, Umapathy C, Musthaq M. Treatment of schizoaffective disorder and schizophrenia with mood symptoms. Am J Psychiatry 1999; 156: 1138-48.

2 Plasky P. Antidepressant usage in schizophrenia. Schizophr Bull 1991: 17:649-657.

3 Siris SG. Diagnosis of secondary depression in schizophrenia. Schizophr Bull 1991; 17: 75-97.

4 Tollefson GD, Sanger TM, Lu Y, Thieme ME. Depressive signs and symptoms in schizophrenia: a prospective blinded 55:250-8.
Sources of funding: Supported by the Clinical Effectiveness Support Unit of the Welsh Office.

Correspondence to: Professor Glyn Lewis, University of Bristol, Cotham House, Cotham Hill, Bristol, BS6 6JL 